\title{
The influence of different workload trainings on some blood parameters in show jumping horses
}

\author{
Robert Kupczyński1 ${ }^{\star *}$, Kinga Śpitalniak ${ }^{1}$, Anna Zwyrzykowska-Wodzińska ${ }^{1}$, \\ and Maria Soroko ${ }^{2}$ \\ ${ }^{I}$ Department of Environment Hygiene and Animal Welfare, Wroclaw University of Environmental and Life \\ Sciences, Wroclaw, Poland \\ ${ }^{2}$ Department of Horse Breeding and Equestrian Studies, Wroclaw University of Environmental and Life \\ Sciences, Wroclaw, Poland
}

KUPCZYŃSKI, R., K, ŚPITALNIAK, A. ZWYRZYKOWSKA-WODZIŃSKA, M. SOROKO: The influence of different workload trainings on some blood parameters in show jumping horses. Vet. arhiv 88, 279-293, 2018.

ABSTRACT

The aim of the study was to assess the changes in the hematology and blood chemistry of jumping horses under the different workload trainings. Group A was trained with a rider and without additional exercises. Group B had an additional daily training session on the treadmill. Blood samples were taken at rest, before exercise and 24 hours after the jumping competition (at the beginning of the season, in the middle and at the end). Regardless of the type of training intensity level and workout load, the post-exercise studies showed an increase in RBC, HGB and HCT. In Group B, there was increase $(\mathrm{P}<0.05)$ of resting parameters RBC and HGB between the start of the season and in the middle. During the study there was an increase in AST, LDH, CK in both groups after training. Horses from Group B, subjected to additional training at the end of the season, had lower activity in the resting parameters of AST, LDH and CK than horses from group A. In group B the resting value of LA was lower in the middle and at the end of the sports season, in comparison to group A. The research indicated the desirability of introducing additional stimulation of movement in horses between the relevant trainings involving a rider.

Key words: show jumping horses; training methods; blood parameters; treadmill

\section{Introduction}

Show jumping is one of the most popular equestrian events. Preparing horses is carried out by inducing the physiological adaptations necessary to compete at a high level, with a minimal risk of injury, and ensuring appropriate behavioral and psychological

\footnotetext{
${ }^{*}$ Corresponding author:

Robert Kupczyński, Department of Environment Hygiene and Animal Welfare, Wroclaw University of Environmental and Life Sciences, Chelmonskiego 38 c, 51-630 Wroclaw, Poland, Phone: +48 723205 941; Fax: +48 723205 866; E-mail: robert. kupczynski@upwr.edu.pl
}

ISSN 0372-5480

Printed in Croatia 
R. Kupczyński et al.: The influence of different workload trainings on some blood parameters in show jumping horses

factors (REED et al., 2004; FERRAZ et al., 2010). Regular training aims at adapting the horse's body system to the changes occurring during intensive physical effort (TRIGO et al., 2010; FAZIO et al., 2011). Properly planned training is necessary to obtain good results. Along with the degree of training, it should be followed by the intensification of exercise that causes the physiological changes needed to achieve optimal performance (ASSENZA et al., 2015).

The methods which allow evaluation of the horse's progress in training involve, amongst other things, analysis of physiological indices, such as cardiac stress tests, as well as examination of hematological and biochemical parameters (PICCIONE et al., 2007; SOARES et al., 2011; FAZIO et al., 2014; KUPCZYŃSKI and ŚPITALNIAK, 2015). These reflect the metabolic and functional processes involved in adaptation to a particular discipline (PICCIONE et al., 2007; SOARES et al., 2011). The results obtained from the study are useful for evaluating the current health performance and the degree of adaptation to intensification of exercise, providing the ability to alter the training program in order to achieve the desired sport performance (CASELLA et al., 2012; ADAMU et al., 2013). Some authors have presented new and useful information concerning the blood changes that take place in response to physical exercise, which will make it possible to evaluate the health status and training levels of jumper horses (BIS-WENCEL et al., 2012; FAZIO et al., 2012).

Automatic horse walkers and treadmills are helpful in proper horse training. Their usefulness stems from the easy control of exercise intensity. During training, the speed and duration of each stage of the exercise are highly reproducible. There is also easy access to horse at the appropriate moments during and after each exercise, for measurement of cardio-pulmonary capacity or blood sampling, and this training develops the aerobic abilities of the horse (OHMURA et al., 2013). Another method of training is aqua-training, previously used for rehabilitation purposes, which currently is used in horse training. This method increases the average aerobic load for horses, as well as helping to maintain correct posture, balance, muscle development, and it also improves the functioning of the circulatory system. This kind of exercise does not lead to any overload of the metabolism and thus has a positive effect on the relevant horse performance (NANKERVIS and WILLIAMS, 2006; LINDNER et al., 2009; LINDNER et al., 2012). The classic forms of providing horses with movement are practicing on a lunge and enabling them to have access to a paddock. This method is the most widespread and has been used in various forms for many years.

The aim of the study was to assess the changes in hematology and blood chemistry in jumping horses trained at different training intensity levels between the relevant trainings and involving a rider. 
R. Kupczyński et al.: The influence of different workload trainings on some blood parameters in show jumping horses

\section{Materials and methods}

The study was conducted on 20 show jumping horses of various breeds, ages, body mass and level of performance. The study was performed in two equestrian sport stables located in the western part of Poland, from March to September 2015. Horses were divided into two groups: "group A" and "group B". Group A comprised of ten horses aged between 5 to 10 years (five geldings and five mares). Group B comprised 10 horses aged between 5 to 10 years (five geldings and 5 mares). The horses used in the study were of the following breeds: Polish Halfbred, Oldenburg, Dutch, and French saddle horse. Both the groups had a similar number of breeds. The animals in the two groups were subjected to different training protocols. Group A had less intensive training than horses from Group B, which performed daily exercises on a treadmill (Horse Gym $2000 \mathrm{GmbH}$, Germany). The detailed weekly training program for the 2 groups is presented in Table 1 .

Table 1. The detailed weekly training program for group A and group B

\begin{tabular}{|l|l|l|}
\hline Weekly training program & \multicolumn{1}{|c|}{ Group A } & \multicolumn{1}{c|}{ Group B } \\
\hline $\begin{array}{l}\text { Dressage training } \\
\text { four times a week }\end{array}$ & $\begin{array}{l}10 \text { min walk, 10 min - relaxed } \\
\text { trot, 5 min - working trot, 5-7 min } \\
\text { gallop, 5-7 min trot, 10 min walk }\end{array}$ & $\begin{array}{l}10 \text { min walk, 10 min relaxed } \\
\text { trot, 5 min working trot, 5-7 min } \\
\text { gallop, 5-7 min trot, 10 min walk }\end{array}$ \\
\hline $\begin{array}{l}\text { Jumping training } \\
\text { twice a week }\end{array}$ & $\begin{array}{l}10 \text { min walk, 10 min - relaxed } \\
\text { trot, 5 min - working trot and } \\
\text { series of 12-16 jumps, height of } \\
80-120 \mathrm{~cm}\end{array}$ & $\begin{array}{l}10 \text { min walk, 10 min - relaxed } \\
\text { trot, 5 min - working trot and } \\
\text { series of 15-18 jumps, height of } \\
100-140 \mathrm{~cm}\end{array}$ \\
\hline $\begin{array}{l}\text { Lunge training } \\
\text { once a week }\end{array}$ & 45 min of lunge training & 45 min of lunge training \\
\hline $\begin{array}{l}\text { Treadmill } \\
\text { exercises* }\end{array}$ & Lack of training & $\begin{array}{l}\text { Daily treadmill exercises for 36 } \\
\text { minutes: 7 min - walk, 7 min } \\
\text { walk with inclined treadmill 6\%, } \\
8 \text { min of walk }\end{array}$ \\
\hline
\end{tabular}

*For treadmill exercises a Horse Gym 2000 treadmill was used

The average ration per day per horse in both groups was $5 \mathrm{~kg}$ of hay twice a day, 4 $\mathrm{kg}$ of crushed oats/day (three portions). Additionally the horses from group A received 50 g per day mineral and vitamin supplement (General P), or a combination of vitamin $\mathrm{E}+\mathrm{Se}+$ Lys (Vitamin E, Selenium \& Lysine) in the amount of $40 \mathrm{~g}$ per day, and 100 $\mathrm{mL}$ of rapeseed oil. In the experiment Group B horses, besides oats were given two types of special feed. The feed mixtures used were FibreMix Extra, FibreMix Easy, Horsgran, high energy feed (Main Ring Gold), and conditioning fodder (Conditioning Mix, Alfa A Oil, Beet Alfa Alfa A Original). The feeds listed were used according to the manufacturer's recommendations. The animals were given $150 \mathrm{~g}$ per day Golden Mineral mineral-vitamin supplement. 
In both centers at the beginning (March - series I) in the middle (June - series II) and at the end of the season (September - series II) three series of sampling exercise session were performed. Blood was collected at rest, immediately after training and 24 hours after training. Blood tests were always performed a week before a competition. Blood was taken from the v. jugularis externa using sterile Sarsted tubes for serum (10 $\mathrm{mL})$ and with an anticoagulant - EDTA $(2 \mathrm{~mL})$. Analyses of hematological parameters were performed using an $\mathrm{ABC}$ Vet (Horiba $\mathrm{ABX}$ Diagnostics, France) analyzer, taking into account parameters such as: red blood cells (RBC), white blood cells (WBC), platelets (PLT), hemoglobin (HGB), hematocrits (HCT), mean corpuscular volume $(\mathrm{MCV})$, mean corpuscular hemoglobin $(\mathrm{MCH})$ and mean corpuscular hemoglobin concentration (MCHC), lymphocytes (LYM), monocytes (MON), and granulocytes (GRA). Biochemical studies were performed using Pentra 400 (Horiba ABX Diagnostics, France). The following parameters were estimated: glucose oxidase method, HORIBA ABX reagents (Glucose PAP, Cat. No. A11A016979); lactic acid (LA) by colorimetric method, HORIBA ABX reagents (Cat. No. A11A01721); aspartate aminotransferase (AST), lactate dehydrogenase (LDH) and creatine kinase (CK) activity to enzyme kinetic method, HORIBA ABX reagents (Cat. No. A11A01629, A11A01824, A11A01632); creatinine by enzymatic method (Cat. No. A11A01907); and Na, K, Cl, using an ionselective adapter in the Pentra 400 analyzer.

The results of the laboratory tests were expressed in SI units, and subjected to statistical analysis by calculating the arithmetic mean and standard deviation. Distribution variables were evaluated using the Shapiro-Wilk test. The data obtained were subjected to analysis of variance (ANOVA). The significance of differences between the test results was determined by Duncan's test. Effects were considered significant at a probability of $\mathrm{P}<0.05$. All statistical analyses were conducted using Statistica v. 10.0.

\section{Results}

The mean values of blood hematological parameters of horses from groups A and $\mathrm{B}$ are presented in Table 2 and 3. In group A, the resting values of WBC, RBC and HGB do not differ significantly throughout the training season. In Group B, we observed a gradual increase $(\mathrm{P}<0.05)$ in the resting values of $\mathrm{RBC}$ between series II and III of the research and in HGB parameter between terms of research I and III. In the series I studies, the HGB and HCT increased significantly $(\mathrm{P}<0.05)$ before and immediately after exercise, in group $\mathrm{B}$ during the study period. In the subsequent periods of research (both in the second series as well as in series III) the RBC, HGB, HCT parameters increased significantly in group $\mathrm{B}$ after training $(\mathrm{P}<0.01)$. An increase of $\mathrm{RBC}$ after training was reported in group $\mathrm{A}$ only at the end of the season (series III). In both groups, no changes in WBC after training were confirmed statistically. In group A, the percentage of GRA increased $(\mathrm{P}<0.01)$ in the second sampling. In the second series of studies the content of LYM increased $(\mathrm{P}<0.05)$ after training in both groups. 
R. Kupczyński et al.: The influence of different workload trainings on some blood parameters in show jumping horses

Table 2. Mean \pm SD values of blood hematological parameters of horses (group A)

\begin{tabular}{|c|c|c|c|c|c|c|}
\hline \multirow[b]{2}{*}{ Item } & \multirow{2}{*}{$\begin{array}{c}\text { Exercise } \\
\text { session }\end{array}$} & \multicolumn{3}{|c|}{ Timepoint } & \multicolumn{2}{|c|}{ P-value } \\
\hline & & Before training & After training & After $24 \mathrm{~h}$ & ES & $\mathrm{TS}$ \\
\hline $\begin{array}{l}\text { WBC } \\
\left(\times 10^{9} / \mathrm{L}\right)\end{array}$ & $\begin{array}{c}\text { I } \\
\text { II } \\
\text { III }\end{array}$ & $\begin{array}{l}6.01 \pm 0.69 \\
6.10 \pm 0.78 \\
6.29 \pm 1.06\end{array}$ & $\begin{array}{l}5.92 \pm 0.76^{\mathrm{a}} \\
6.67 \pm 0.83^{\mathrm{b}} \\
6.88 \pm 1.04^{\mathrm{b}}\end{array}$ & $\begin{array}{l}6.12 \pm 0.23 \\
6.24 \pm 0.56 \\
6.20 \pm 0.13\end{array}$ & 0.01 & 0.48 \\
\hline $\begin{array}{l}\text { RBC } \\
\left(\times 10^{12} / \mathrm{L}\right)\end{array}$ & $\begin{array}{c}\text { I } \\
\text { II } \\
\text { III }\end{array}$ & $\begin{array}{l}7.56 \pm 0.47 \\
7.42 \pm 0.71 \\
7.49 \pm 0.42\end{array}$ & $\begin{array}{l}7.67 \pm 0.49^{\mathrm{A}} \\
8.01 \pm 0.60 \\
8.37 \pm 0.54^{\mathrm{B}}\end{array}$ & $\begin{array}{l}7.55 \pm 0.34 \\
7.65 \pm 0.53 \\
8.12 \pm 0.23\end{array}$ & 0.03 & 0.01 \\
\hline $\begin{array}{l}\mathrm{HGB} \\
(\mathrm{mmol} / \mathrm{L})\end{array}$ & $\begin{array}{c}\text { I } \\
\text { II } \\
\text { III } \\
\end{array}$ & $\begin{array}{l}7.47 \pm 1.50 \\
7.50 \pm 0.98 \\
7.63 \pm 0.63 \\
\end{array}$ & $\begin{array}{l}7.57 \pm 0.71^{\mathrm{a}} \\
8.17 \pm 0.98 \\
8.56 \pm 0.82 \mathrm{~b}\end{array}$ & $\begin{array}{l}7.35 \pm 0.15 \\
7.45 \pm 0.83 \\
8.03 \pm 0.33 \\
\end{array}$ & 0.24 & 0.01 \\
\hline $\begin{array}{l}\mathrm{HCT} \\
(\mathrm{L} / \mathrm{L})\end{array}$ & $\begin{array}{c}\text { I } \\
\text { II } \\
\text { III } \\
\end{array}$ & $\begin{array}{l}0.36 \pm 0.04^{\mathrm{a}} \\
0.33 \pm 0.04 \\
0.34 \pm 0.03^{\mathrm{b}}\end{array}$ & $\begin{array}{l}0.38 \pm 0.03 \\
0.37 \pm 0.04 \\
0.38 \pm 0.04\end{array}$ & $\begin{array}{l}0.35 \pm 0.02 \\
0.35 \pm 0.05 \\
0.36 \pm 0.01\end{array}$ & 0.02 & 0.01 \\
\hline $\begin{array}{l}\text { PLT } \\
\left(\times 10^{9} / \mathrm{L}\right)\end{array}$ & $\begin{array}{c}\text { I } \\
\text { II } \\
\text { III } \\
\end{array}$ & $\begin{array}{l}151.57 \pm 9.83^{\mathrm{a}} \\
134.86 \pm 14.71 \\
115.56 \pm 6.94^{\mathrm{b}}\end{array}$ & $\begin{array}{l}181.71 \pm 75.13 \\
141.67 \pm 67.23 \\
124.38 \pm 23.14\end{array}$ & $\begin{array}{l}167.34 \pm 34.23 \\
136.44 \pm 54.2 \\
120.76 \pm 14.98\end{array}$ & 0.01 & 0.45 \\
\hline $\begin{array}{l}\text { MCV } \\
(\mathrm{fL})\end{array}$ & $\begin{array}{c}\text { I } \\
\text { II } \\
\text { III } \\
\end{array}$ & $\begin{array}{l}48.15 \pm 3.98 \\
45.00 \pm 3.32 \\
45.00 \pm 2.92 \\
\end{array}$ & $\begin{array}{l}48.71 \pm 3.30 \\
46.17 \pm 3.19 \\
45.25 \pm 3.24\end{array}$ & $\begin{array}{l}48.34 \pm 4.96 \\
45.35 \pm 3.2 \\
45.12 \pm 0.98\end{array}$ & 0.01 & 0.99 \\
\hline $\begin{array}{l}\mathrm{MCH} \\
(\mathrm{fmoL})\end{array}$ & $\begin{array}{c}\text { I } \\
\text { II } \\
\text { III } \\
\end{array}$ & $\begin{array}{l}0.96 \pm 0.17 \\
1.01 \pm 0.08 \\
1.02 \pm 0.07 \\
\end{array}$ & $\begin{array}{l}0.96 \pm 0.13 \\
1.02 \pm 0.08 \\
1.02 \pm 0.07\end{array}$ & $\begin{array}{r}0.96 \pm 0.04 \\
1.0 \pm 0.05 \\
1.02 \pm 0.07\end{array}$ & 0.07 & 0.99 \\
\hline $\begin{array}{l}\mathrm{MCHC} \\
(\mathrm{mmol} / \mathrm{L})\end{array}$ & $\begin{array}{c}\text { I } \\
\text { II } \\
\text { III } \\
\end{array}$ & $\begin{array}{l}19.70 \pm 2.70^{\mathrm{aA}} \\
22.37 \pm 0.17^{\mathrm{b}} \\
22.68 \pm 0.25^{\mathrm{aB}}\end{array}$ & $\begin{array}{l}20.16 \pm 1.51^{\mathrm{aA}} \\
22.05 \pm 0.35^{\mathrm{bA}} \\
22.65 \pm 0.19^{\mathrm{B}}\end{array}$ & $\begin{array}{l}19.98 \pm 0.67 \\
22.28 \pm 0.56 \\
22.59 \pm 0.24\end{array}$ & 0.01 & 0.73 \\
\hline $\begin{array}{l}\text { GRA } \\
(\%)\end{array}$ & $\begin{array}{c}\text { I } \\
\text { II } \\
\text { III } \\
\end{array}$ & $\begin{array}{l}61.47 \pm 5.85^{\mathrm{A}} \\
70.83 \pm 2.69^{\mathrm{aB}} \\
63.47 \pm 6.07^{\mathrm{b}}\end{array}$ & $\begin{array}{l}58.51 \pm 7.87^{\mathrm{a}} \\
67.37 \pm 2.39^{\mathrm{b}} \\
65.11 \pm 4.63^{\mathrm{a}}\end{array}$ & $\begin{array}{l}58.23 \pm 6.35 \\
70.03 \pm 3.43 \\
65.10 \pm 4.32\end{array}$ & 0.01 & 0.81 \\
\hline $\begin{array}{l}\text { LYM } \\
(\%)\end{array}$ & $\begin{array}{c}\text { I } \\
\text { II } \\
\text { III } \\
\end{array}$ & $\begin{array}{l}29.44 \pm 4.45^{\mathrm{a}} \\
23.89 \pm 2.68^{\mathrm{b}} \\
27.71 \pm 4.57^{\mathrm{a}}\end{array}$ & $\begin{array}{l}31.96 \pm 5.06 \\
27.37 \pm 2.37 \\
27.59 \pm 4.11\end{array}$ & $\begin{array}{l}29.89 \pm 3.25 \\
25.35 \pm 1.45 \\
27.43 \pm 3.21^{\mathrm{a}}\end{array}$ & 0.18 & 0.06 \\
\hline $\begin{array}{l}\text { MON } \\
(\%)\end{array}$ & $\begin{array}{c}\text { I } \\
\text { II } \\
\text { III }\end{array}$ & $\begin{array}{l}9.32 \pm 2.48^{\mathrm{A}} \\
5.29 \pm 0.66^{\mathrm{B}} \\
8.82 \pm 2.20^{\mathrm{A}}\end{array}$ & $\begin{array}{l}9.53 \pm 3.50^{\mathrm{a}} \\
5.27 \pm 0.60^{\mathrm{b}} \\
7.38 \pm 1.91^{\mathrm{a}}\end{array}$ & $\begin{array}{l}9.41 \pm 1.13 \\
5.26 \pm 2.34 \\
7.30 \pm 3.41\end{array}$ & 0.03 & 0.87 \\
\hline
\end{tabular}

ES - significant differences between exercise sessions; TS - significant differences between times of blood sample collection; A, B - significant differences between exercise session, $\mathrm{P}<0.01 ; \mathrm{a}, \mathrm{b}$ - significant differences between exercise sessions in sampling blood before or after training, $\mathrm{P}<0.05$ 
R. Kupczyński et al.: The influence of different workload trainings on some blood parameters in show jumping horses

Table 3. Mean \pm SD values of blood hematological parameters of horses (group B)

\begin{tabular}{|c|c|c|c|c|c|c|}
\hline \multirow[b]{2}{*}{ Item } & \multirow{2}{*}{$\begin{array}{c}\text { Exercise } \\
\text { session }\end{array}$} & \multicolumn{3}{|c|}{ Timepoint } & \multicolumn{2}{|c|}{ P-value } \\
\hline & & Before training & After training & After $24 \mathrm{~h}$ & ES & $\mathrm{TS}$ \\
\hline \multirow{3}{*}{$\begin{array}{l}\text { WBC } \\
\left(\times 10^{9} / \mathrm{L}\right)\end{array}$} & I & $6.74 \pm 1.32$ & $7.84 \pm 1.63$ & $6.89 \pm 0.43$ & \multirow{3}{*}{0.02} & \multirow{3}{*}{0.12} \\
\hline & II & $6.44 \pm 1.27$ & $7.52 \pm 2.10$ & $6.49 \pm 0.67$ & & \\
\hline & III & $7.06 \pm 1.56$ & $7.39 \pm 1.47$ & $6.87 \pm 0.34$ & & \\
\hline \multirow{3}{*}{$\begin{array}{l}\mathrm{RBC} \\
\left(\times 10^{12} / \mathrm{L}\right)\end{array}$} & I & $7.67 \pm 0.62^{\mathrm{a}}$ & $8.38 \pm 0.81^{\mathrm{a}}$ & $7.89 \pm 0.76$ & \multirow{3}{*}{0.50} & \multirow{3}{*}{0.01} \\
\hline & II & $7.64 \pm 0.41^{\mathrm{a}}$ & $9.01 \pm 0.23$ & $8.15 \pm 0.34$ & & \\
\hline & III & $8.30 \pm 0.39^{\mathrm{b}}$ & $9.17 \pm 0.53^{b}$ & $8.33 \pm 0.12$ & & \\
\hline \multirow{3}{*}{$\begin{array}{l}\mathrm{HGB} \\
(\mathrm{mmol} / \mathrm{L})\end{array}$} & I & $7.35 \pm 0.47^{\mathrm{a}}$ & $7.99 \pm 0.68^{\mathrm{a}}$ & $7.55 \pm 0.24$ & \multirow{3}{*}{0.18} & \multirow{3}{*}{0.01} \\
\hline & II & $7.29 \pm 0.48$ & $8.50 \pm 0.58$ & $7.91 \pm 0.45$ & & \\
\hline & III & $7.87 \pm 0.46^{\mathrm{b}}$ & $8.74 \pm 0.55^{\mathrm{b}}$ & $8.14 \pm 0.53$ & & \\
\hline \multirow{3}{*}{$\begin{array}{l}\mathrm{HCT} \\
(\mathrm{L} / \mathrm{L})\end{array}$} & I & $0.34 \pm 0.03^{\mathrm{a}}$ & $0.39 \pm 0.03$ & $035 \pm 0.12$ & \multirow{3}{*}{0.09} & \multirow{3}{*}{0.01} \\
\hline & II & $0.32 \pm 0.02^{\mathrm{b}}$ & $0.38 \pm 0.03$ & $0.34 \pm 0.07$ & & \\
\hline & III & $0.36 \pm 0.02^{\mathrm{a}}$ & $0.40 \pm 0.03$ & $0.37 \pm 0.02$ & & \\
\hline \multirow{3}{*}{$\begin{array}{l}\text { PLT } \\
\left(\times 10^{9} / \mathrm{L}\right)\end{array}$} & I & $177.50 \pm 61.60$ & $196.7 \pm 84.25$ & $190.2 \pm 47.8$ & \multirow{3}{*}{0.01} & \multirow{3}{*}{0.48} \\
\hline & II & $143.00 \pm 29.08$ & $152.80 \pm 30.50$ & $148.78 \pm 28.5$ & & \\
\hline & III & $239.29 \pm 39.81$ & $164.86 \pm 37.91$ & $119.99 \pm 56.8$ & & \\
\hline \multirow{3}{*}{$\begin{array}{l}\text { MCV } \\
(\mathrm{fL})\end{array}$} & I & $46.75 \pm 2.60^{\mathrm{a}}$ & $47.00 \pm 2.78^{a}$ & $46.64 \pm 1.15$ & \multirow{3}{*}{0.05} & \multirow{3}{*}{0.8} \\
\hline & II & $42.43 \pm 2.83^{b}$ & $42.40 \pm 2.07^{\mathrm{A}}$ & $41.98 \pm 2.45$ & & \\
\hline & III & $43.57 \pm 2.64^{b}$ & $43.67 \pm 2.14^{\mathrm{b}}$ & $43.54 \pm 0.87$ & & \\
\hline \multirow{3}{*}{$\begin{array}{l}\mathrm{MCH} \\
(\mathrm{fmoL})\end{array}$} & I & $0.96 \pm 0.06$ & $0.96 \pm 0.07$ & $0.96 \pm 0.03$ & \multirow{3}{*}{0.01} & \multirow{3}{*}{0.97} \\
\hline & II & $0.96 \pm 0.06$ & $0.94 \pm 0.04^{b}$ & $0.95 \pm 0.12$ & & \\
\hline & III & $0.95 \pm 0.06$ & $0.96 \pm 0.06$ & $0.96 \pm 0.03$ & & \\
\hline \multirow{3}{*}{$\begin{array}{l}\mathrm{MCHC} \\
(\mathrm{mmol} / \mathrm{L})\end{array}$} & I & $20.51 \pm 0.51^{\mathrm{A}}$ & $20.35 \pm 0.38^{\mathrm{A}}$ & $20.45 \pm 0.89$ & \multirow{3}{*}{0.01} & \multirow{3}{*}{0.15} \\
\hline & II & $22.51 \pm 0.33^{\mathrm{B}}$ & $22.30 \pm 0.39^{\mathrm{B}}$ & $22.45 \pm 0.35$ & & \\
\hline & III & $21.79 \pm 0.13^{\mathrm{B}}$ & $21.87 \pm 0.22^{\mathrm{A}}$ & $21.85 \pm 0.56$ & & \\
\hline & I & $74.45 \pm 5.07$ & $73.35 \pm 4.56$ & $73 \pm 4.67$ & & \\
\hline GRA & II & $71.53 \pm 6.56$ & $68.08 \pm 3.83$ & $67.54 \pm 8.23$ & 0.01 & 0.24 \\
\hline & III & $70.92 \pm 7.05$ & $69.61 \pm 8.33$ & $69.01 \pm 0.87$ & & \\
\hline & I & $19.28 \pm 5.00$ & $20.63 \pm 4.74$ & $20.01 \pm 1.24$ & & \\
\hline $\begin{array}{l}\text { LYM } \\
(0 / 9)\end{array}$ & II & $22.17 \pm 5.28$ & $25.52 \pm 2.46$ & $23.42 \pm 4.03$ & 0.01 & 0.01 \\
\hline & III & $21.19 \pm 5.40$ & $23.19 \pm 6.28$ & $22.09 \pm 2.57$ & & \\
\hline & I & $6.24 \pm 0.77^{\mathrm{a}}$ & $6.33 \pm 0.80$ & $6.01 \pm 2.45$ & & \\
\hline MON & II & $6.30 \pm 1.75$ & $6.40 \pm 1.67$ & $6.34 \pm 1.77$ & 0.01 & 0.91 \\
\hline$(\%)$ & III & $7.93 \pm 1.99^{b}$ & $7.99 \pm 2.24$ & $7.89 \pm 0.63$ & & \\
\hline
\end{tabular}

ES - significant differences between exercise sessions; TS - significant differences between times of blood sample collection; A, B - significant differences between exercise sessions, $\mathrm{P}<0.01$; $\mathrm{a}, \mathrm{b}$ - significant differences between exercise sessions in sampling blood before or after training, $\mathrm{P}<0.05$ 
R. Kupczyński et al.: The influence of different workload trainings on some blood parameters in show jumping horses

Table 4. Mean \pm SD values of blood biochemical parameters of horses (group A)

\begin{tabular}{|c|c|c|c|c|c|c|}
\hline \multirow[b]{2}{*}{ Item } & \multirow{2}{*}{$\begin{array}{l}\text { Exercise } \\
\text { session }\end{array}$} & \multicolumn{3}{|c|}{ Timepoint } & \multicolumn{2}{|c|}{ P-value } \\
\hline & & Before training & After training & After $24 \mathrm{~h}$ & ES & TS \\
\hline $\begin{array}{l}\text { AST } \\
(\mathrm{U} / \mathrm{L})\end{array}$ & $\begin{array}{c}\text { I } \\
\text { II } \\
\text { III }\end{array}$ & $\begin{array}{l}286.97 \pm 29.39^{\mathrm{a}} \\
240.46 \pm 92.73^{\mathrm{b}} \\
284.50 \pm 43.98^{\mathrm{a}}\end{array}$ & $\begin{array}{l}296.57 \pm 25.60^{\mathrm{a}} \\
250.49 \pm 97.05^{\mathrm{b}} \\
294.49 \pm 49.64\end{array}$ & $\begin{aligned} & 276.54 \pm 35.65 \\
& 245.91 \pm 39.05 \\
& 287.9 \pm 59.62 \\
&\end{aligned}$ & 0.01 & 0.71 \\
\hline $\begin{array}{l}\text { LDH } \\
(\mathrm{U} / \mathrm{L})\end{array}$ & $\begin{array}{c}\text { I } \\
\text { II } \\
\text { III } \\
\end{array}$ & $\begin{array}{l}520.59 \pm 148.76^{\mathrm{A}} \\
459.69 \pm 214.99^{\mathrm{a}} \\
645.63 \pm 83.35 b^{\mathrm{B}}\end{array}$ & $\begin{array}{l}552.73 \pm 158.89^{\mathrm{aA}} \\
494.21227 .75^{\mathrm{b}} \\
665.35 \pm 62.75^{\mathrm{B}}\end{array}$ & $\begin{array}{l}532.73 \pm 118.07 \\
448.61 \pm 177.07^{\mathrm{A}} \\
657.35 \pm 72.75^{\mathrm{B}} \\
\end{array}$ & 0.01 & 0.51 \\
\hline $\begin{array}{l}\mathrm{CK} \\
(\mathrm{U} / \mathrm{L})\end{array}$ & $\begin{array}{c}\text { I } \\
\text { II } \\
\text { III } \\
\end{array}$ & $\begin{array}{l}198.50 \pm 30.92 \\
187.81 \pm 65.41 \\
207.16 \pm 39.11 \\
\end{array}$ & $\begin{array}{l}296.57 \pm 25.60^{\mathrm{A}} \\
201.16 \pm 83.67^{\mathrm{B}} \\
218.59 \pm 42.49^{\mathrm{B}}\end{array}$ & $\begin{array}{l}209.03 \pm 25.09^{\mathrm{A}} \\
198.36 \pm 63.67^{\mathrm{B}} \\
201.59 \pm 42.49^{\mathrm{B}} \\
\end{array}$ & 0.01 & 0.61 \\
\hline $\begin{array}{l}\text { GLU } \\
(\mathrm{mmol} / \mathrm{L})\end{array}$ & $\begin{array}{c}\text { I } \\
\text { II } \\
\text { III } \\
\end{array}$ & $\begin{array}{l}5.68 \pm 0.25^{\mathrm{A}} \\
4.41 \pm 0.87^{\mathrm{B}} \\
4.87 \pm 0.42^{\mathrm{B}} \\
\end{array}$ & $\begin{array}{l}5.93 \pm 0.79^{\mathrm{A}} \\
4.45 \pm 1.03^{\mathrm{B}} \\
5.05 \pm 0.61^{\mathrm{B}} \\
\end{array}$ & $\begin{array}{l}4.90 \pm 0.86^{\mathrm{B}} \\
4.31 \pm 0.03^{\mathrm{B}} \\
3.90 \pm 0.61^{\mathrm{A}} \\
\end{array}$ & 0.01 & 0.01 \\
\hline $\begin{array}{l}\text { Creatinine } \\
(\mu \mathrm{mol} / \mathrm{L})\end{array}$ & $\begin{array}{c}\text { I } \\
\text { II } \\
\text { III } \\
\end{array}$ & $\begin{aligned} & 107.95 \pm 6.64^{\mathrm{a}} \\
& 90.81 \pm 18.81^{\mathrm{b}} \\
& 113.96 \pm 18.01^{\mathrm{a}} \\
&\end{aligned}$ & $\begin{aligned} 120.83 & \pm 15.77^{\mathrm{A}} \\
93.40 & \pm 23.57^{\mathrm{B}} \\
124.99 & \pm 7.50^{\mathrm{C}}\end{aligned}$ & $\begin{aligned} & 118.73 \pm 25.07^{\mathrm{A}} \\
& 91.84 \pm 13.43^{\mathrm{B}} \\
& 120.09 \pm 5.20^{\mathrm{C}} \\
&\end{aligned}$ & 0.01 & 0.11 \\
\hline $\begin{array}{l}\mathrm{LA} \\
(\mathrm{mmol} / \mathrm{L})\end{array}$ & $\begin{array}{c}\text { I } \\
\text { II } \\
\text { III } \\
\end{array}$ & $\begin{array}{l}0.81 \pm 0.03^{\mathrm{A}} \\
1.43 \pm 0.41^{\mathrm{B}} \\
1.23 \pm 0.13 \\
\end{array}$ & $\begin{array}{l}1.97 \pm 0.15^{\mathrm{A}} \\
2.40 \pm 0.26^{\mathrm{B}} \\
2.34 \pm 0.28^{\mathrm{B}} \\
\end{array}$ & $\begin{array}{l}1.16 \pm 0.85^{\mathrm{A}} \\
1.90 \pm 0.64 \\
2.03 \pm 0.08^{\mathrm{B}} \\
\end{array}$ & 0.01 & 0.60 \\
\hline $\begin{array}{l}\mathrm{Na} \\
(\mathrm{mmol} / \mathrm{L})\end{array}$ & $\begin{array}{c}\text { I } \\
\text { II } \\
\text { III } \\
\end{array}$ & $\begin{array}{l}131.03 \pm 2.38^{\mathrm{A}} \\
105.59 \pm 20.15^{\mathrm{B}} \\
133.73 \pm 0.68^{\mathrm{C}} \\
\end{array}$ & $\begin{array}{l}134.51 \pm 1.76^{\mathrm{A}} \\
106.20 \pm 19.12^{\mathrm{B}} \\
134.64 \pm 0.83^{\mathrm{A}} \\
\end{array}$ & $\begin{array}{l}132.11 \pm 1.05^{\mathrm{A}} \\
106.01 \pm 13.02^{\mathrm{B}} \\
134.01 \pm 0.73^{\mathrm{A}} \\
\end{array}$ & 0.01 & 0.85 \\
\hline $\begin{array}{l}\mathrm{K} \\
(\mathrm{mmol} / \mathrm{L})\end{array}$ & $\begin{array}{c}\text { I } \\
\text { II } \\
\text { III } \\
\end{array}$ & $\begin{array}{l}3.02 \pm 0.81^{\mathrm{a}} \\
2.61 \pm 0.57^{\mathrm{a}} \\
3.73 \pm 0.29^{\mathrm{b}} \\
\end{array}$ & $\begin{array}{l}3.83 \pm 0.47 \\
3.19 \pm 0.72^{\mathrm{A}} \\
4.06 \pm 0.22^{\mathrm{B}} \\
\end{array}$ & $\begin{array}{l}3.45 \pm 0.07 \\
2.99 \pm 0.82^{\mathrm{A}} \\
4.87 \pm 0.12^{\mathrm{B}} \\
\end{array}$ & 0.01 & 0.01 \\
\hline $\begin{array}{l}\mathrm{Cl} \\
(\mathrm{mmol} / \mathrm{L})\end{array}$ & $\begin{array}{c}\text { I } \\
\text { II } \\
\text { III }\end{array}$ & $\begin{array}{l}97.97 \pm 1.21 \\
90.44 \pm 13.81 \\
98.90 \pm 1.52\end{array}$ & $\begin{array}{l}95.53 \pm 2.32 \\
80.43 \pm 11.51 \\
95.33 \pm 1.60\end{array}$ & $\begin{array}{r}96.73 \pm 1.36 \\
83.7 \pm 3.01 \\
97.13 \pm 1.02\end{array}$ & 0.01 & 0.99 \\
\hline
\end{tabular}

ES - significant differences between exercise sessions; TS - significant differences between times of blood sample collection; A, B, C - significant differences between exercise sessions, $\mathrm{P}<0.01$; $\mathrm{a}$, b - significant differences between exercise sessions in sampling blood before or after training, $\mathrm{P}<0.05$ 
R. Kupczyński et al.: The influence of different workload trainings on some blood parameters in show jumping horses

Table 5. Mean \pm SD values of blood biochemical parameters of horses (group B)

\begin{tabular}{|c|c|c|c|c|c|c|}
\hline \multirow[b]{2}{*}{ Item } & \multirow{2}{*}{$\begin{array}{c}\text { Exercise } \\
\text { session }\end{array}$} & \multicolumn{3}{|c|}{ Timepoint } & \multicolumn{2}{|c|}{$\mathrm{P}$-value } \\
\hline & & Before training & After training & After $24 \mathrm{~h}$ & ES & $\mathrm{TS}$ \\
\hline $\begin{array}{l}\mathrm{AST} \\
(\mathrm{U} / \mathrm{L})\end{array}$ & \begin{tabular}{|c|} 
I \\
II \\
III
\end{tabular} & $\begin{array}{l}216.39 \pm 49.77^{\mathrm{a}} \\
265.43 \pm 27.09^{\mathrm{b}} \\
259.17 \pm 28.70 \\
\end{array}$ & $\begin{array}{l}257.80 \pm 25.82^{\mathrm{A}} \\
297.22 \pm 26.30^{\mathrm{aB}} \\
265.50 \pm 23.15^{\mathrm{b}}\end{array}$ & $\begin{array}{l}227.80 \pm 25.82^{\mathrm{A}} \\
257.12 \pm 12.37^{\mathrm{B}} \\
253.61 \pm 42.15\end{array}$ & 0.01 & 0.04 \\
\hline $\begin{array}{l}\mathrm{LDH} \\
(\mathrm{U} / \mathrm{L})\end{array}$ & $\begin{array}{c}\text { I } \\
\text { II } \\
\text { III } \\
\end{array}$ & $\begin{array}{l}353.55 \pm 104.39^{\mathrm{A}} \\
524.55 \pm 81.56^{\mathrm{B}} \\
513.91 \pm 69.10^{\mathrm{B}}\end{array}$ & $\begin{array}{l}407.20 \pm 86.05^{\mathrm{aA}} \\
641.92 \pm 113.04^{\mathrm{aB}} \\
525.93 \pm 98.63^{\mathrm{b}}\end{array}$ & $\begin{array}{l}378.28 \pm 85.15^{\mathrm{aA}} \\
567.92 \pm 113.04^{\mathrm{aB}} \\
508.24 \pm 34.73^{\mathrm{b}}\end{array}$ & 0.01 & 0.04 \\
\hline $\begin{array}{l}\text { CK } \\
(\mathrm{U} / \mathrm{L})\end{array}$ & $\begin{array}{c}\text { I } \\
\text { II } \\
\text { III }\end{array}$ & $\begin{array}{l}121.81 \pm 32.09^{\mathrm{aA}} \\
180.53 \pm 52.93^{\mathrm{B}} \\
159.94 \pm 29.43^{\mathrm{b}}\end{array}$ & $\begin{array}{l}142.23 \pm 16.38^{\mathrm{A}} \\
200.06 \pm 25.12^{\mathrm{B}} \\
169.40 \pm 25.43^{\mathrm{B}}\end{array}$ & $\begin{array}{l}134.53 \pm 19.08^{\mathrm{aA}} \\
164.56 \pm 15.62^{\mathrm{B}} \\
160.40 \pm 25.43^{\mathrm{b}}\end{array}$ & 0.01 & 0.24 \\
\hline $\begin{array}{l}\text { GLU } \\
(\mathrm{mmol} / \mathrm{L})\end{array}$ & $\begin{array}{c}\text { I } \\
\text { II } \\
\text { III }\end{array}$ & $\begin{array}{l}5.50 \pm 1.09 \\
5.08 \pm 0.66 \\
5.11 \pm 0.52\end{array}$ & $\begin{array}{l}5.74 \pm 0.82 \\
5.78 \pm 0.36 \\
5.83 \pm 0.43\end{array}$ & $\begin{array}{l}5.30 \pm 0.75 \\
5.15 \pm 0.96 \\
4.95 \pm 0.87\end{array}$ & 0.01 & 0.45 \\
\hline $\begin{array}{l}\text { Creatinine } \\
(\mu \mathrm{mol} / \mathrm{L})\end{array}$ & $\begin{array}{c}\text { I } \\
\text { II } \\
\text { III } \\
\end{array}$ & $\begin{aligned} & 92.71 \pm 17.63^{\mathrm{a}} \\
& 97.54 \pm 8.75^{\mathrm{a}} \\
& 115.43 \pm 17.70^{\mathrm{b}} \\
&\end{aligned}$ & $\begin{array}{l}103.24 \pm 10.82^{\mathrm{A}} \\
110.00 \pm 13.37 \\
117.51 \pm 6.17^{\mathrm{B}} \\
\end{array}$ & $\begin{aligned} & 100.98 \pm 17.22^{\mathrm{A}} \\
& 99.07 \pm 20.07 \\
& 112.99 \pm 12.95^{\mathrm{B}} \\
&\end{aligned}$ & 0.01 & 0.04 \\
\hline $\begin{array}{l}\mathrm{LA} \\
(\mathrm{mmol} / \mathrm{L})\end{array}$ & $\begin{array}{c}\text { I } \\
\text { II } \\
\text { III } \\
\end{array}$ & $\begin{array}{l}1.03 \pm 0.17^{\mathrm{aA}} \\
1.35 \pm 0.46^{\mathrm{B}} \\
1.06 \pm 0.30^{\mathrm{b}} \\
\end{array}$ & $\begin{array}{l}2.67 \pm 0.33^{\mathrm{aA}} \\
2.46 \pm 0.50^{\mathrm{B}} \\
2.10 \pm 0.28^{\mathrm{b}} \\
\end{array}$ & $\begin{array}{l}1.06 \pm 1.03^{\mathrm{aA}} \\
1.67 \pm 0.45^{\mathrm{B}} \\
1.29 \pm 0.23^{\mathrm{b}} \\
\end{array}$ & 0.01 & 0.57 \\
\hline $\begin{array}{l}\mathrm{Na} \\
(\mathrm{mmol} / \mathrm{L})\end{array}$ & $\begin{array}{c}\text { I } \\
\text { II } \\
\text { III }\end{array}$ & $\begin{array}{l}118.88 \pm 19.46 \\
130.68 \pm 1.39^{\mathrm{a}} \\
133.10 \pm 2.29^{\mathrm{b}}\end{array}$ & $\begin{array}{l}127.71 \pm 6.04 \\
130.80 \pm 0.55 \\
131.94 \pm 1.36\end{array}$ & $\begin{array}{l}120.11 \pm 4.08 \\
115.09 \pm 0.65 \\
130.94 \pm 1.36\end{array}$ & 0.05 & 0.10 \\
\hline $\begin{array}{l}\mathrm{K} \\
(\mathrm{mmol} / \mathrm{L})\end{array}$ & $\begin{array}{c}\text { I } \\
\text { II } \\
\text { III } \\
\end{array}$ & $\begin{array}{l}3.25 \pm 0.65 \\
3.50 \pm 0.42 \\
3.27 \pm 0.67^{\mathrm{b}} \\
\end{array}$ & $\begin{array}{l}3.77 \pm 0.28 \\
3.95 \pm 0.15 \\
3.89 \pm 0.18 \\
\end{array}$ & $\begin{array}{l}3.56 \pm 0.78 \\
3.75 \pm 0.09 \\
3.29 \pm 0.89 \\
\end{array}$ & 0.33 & 0.01 \\
\hline $\begin{array}{l}\mathrm{Cl} \\
(\mathrm{mmol} / \mathrm{L})\end{array}$ & $\begin{array}{c}\text { I } \\
\text { II } \\
\text { III } \\
\end{array}$ & $\begin{array}{r}89.79 \pm 4.60^{\mathrm{A}} \\
97.19 \pm 0.89^{\mathrm{B}} \\
100.73 \pm 2.85^{\mathrm{C}} \\
\end{array}$ & $\begin{array}{l}96.14 \pm 4.30 \\
94.46 \pm 0.91^{\mathrm{A}} \\
99.46 \pm 0.77^{\mathrm{B}} \\
\end{array}$ & $\begin{array}{c}99.24 \pm 2.06 \\
95.16 \pm 2.11^{\mathrm{A}} \\
100.02 \pm 0.17^{\mathrm{B}} \\
\end{array}$ & 0.01 & 0.27 \\
\hline
\end{tabular}

ES - significant differences between exercise sessions; TS - significant differences between times of blood sample collection; A, B - significant differences between exercise sessions, $\mathrm{P}<0.01$; $\mathrm{a}, \mathrm{b}$ - significant differences between exercise sessions in sampling blood before or after training, $\mathrm{P}<0.05$

In group A, resting values of AST and glucose were the highest in series I, but in the case of LDH, CK, and creatinine in series III (Table 4). In Group B, the value of the resting AST and CK was the highest in the blood collected in the series II tests, and creatinine in the series III tests (Table 5). In both groups, the highest resting concentration of LA was recorded in the mid-season training. An upward trend in the average values of all biochemical parameters was reported after training and then after rest they returned to values closer to the resting ones. This increase was confirmed statistically $(\mathrm{P}<0.01)$ in the first and second series of tests in group B regarding the activity of AST, LDH, and in A group CK activity in the test I series. Similar results were recorded in the series II tests. 
R. Kupczyński et al.: The influence of different workload trainings on some blood parameters in show jumping horses

In the middle of the training period a statistically significant increase in LDH activity was found $(\mathrm{P}<0.01)$ after training in group $\mathrm{A}$, while in Group $\mathrm{B}$, after training there was a significant increase $(\mathrm{P}<0.01)$ in AST, LDH in series I and II tests. In the last series of tests we found an increase in LDH $(\mathrm{P}<0.05)$ after training in group A. During the sporting season, the LA concentration in both groups showed an upward trend $(\mathrm{P}<0.05)$ after a workout and returned to values close to resting ones.

The resting concentrations of $\mathrm{Na}$ and $\mathrm{Cl}$ showed upward trends in the course of the season in group B. In group A a decrease was found in the concentration of resting $\mathrm{Na}, \mathrm{K}$ and $\mathrm{Cl}$ in the middle of the training season. The lowest the resting value of $\mathrm{Na}$ concentration in the blood was reported in the series II tests in Group A. In groups A and $\mathrm{B}$ resting $\mathrm{Cl}$ increased significantly $(\mathrm{P}<0.05)$ at the end of the season. In the case of $\mathrm{K}$, a significant increase was found $(\mathrm{P}<0.05)$ in post-exercise testing in the series III tests postexercise in group A and in the series II and III tests in Group B.

\section{Discussion}

All properly conducted training increases the body's capacity. Examination of the impact of exercise on the formation of various blood parameters can provide practical information about the effectiveness of the training program. According to PICCIONE et al. (2010) adaptive changes in hematological parameters might ensure the efficient transfer of oxygen to the muscles. Regardless of the scale of the effort and the type of exercise, in post-exercise studies an increase may be observed in the number of red blood cells, which is closely related to the increase in HGB and HCT (PICCIONE et al., 2008). A larger quantity of RBC is normal for horses that are trained properly (KUPCZYŃSKI and ŚPITALNIAK, 2015). In our study, a greater increase in RBC was found in group B after exercise. Additionally, at the end of the season of the research in this group higher resting parameters of RBC, HGB, HTC were observed. Hematological changes are known to result from contraction of the spleen, which in turn is a consequence of the physical effort. Another factor stimulating the function of the spleen and an increase in RBC is stress (PICCIONE et al., 2008; PICCIONE et al., 2010). The increase in (epinephrine like) catecholamines observed in stressful situations stimulates the ejection of blood cells (ASSENZA et al., 2013). The return to resting values observed in our study (in both groups) is an indicator of proper preparation for exercise.

The decrease in MCV values after exercise observed in this study has also been found by several other authors (MUÑOZ et al., 2002; KUPCZYŃSKI and ŚPITLNIAK, 2015). The decreased MCV was the result of intensive effort, along with increased catecholamine concentrations (MUÑOZ et al., 2002). The increase in WBC after exercise may be caused by the release of large numbers of lymphocytes into the circulation from the spleen (PICCIONE et al., 2010; ZOBBA et al., 2011; ASSENZA et al., 2013). During the 
contraction of the spleen PLT are released into the bloodstream, according to ASSENZA et al. (2015) and they cause an increase in post-exercise research. In the early stages of training, the number of PLT may reach extremely high values.

The highest value of PLT was observed in group B at the end of the season. Both trainings associated with an increase in parameters such as RBC, HGB, HCT and PLT, are needed for the proper functioning of muscles, to increase the aerobic capacity of the organism, and mitigate the effects of stress associated with physical effort. However, in the case of PLT high levels that last excessively long after exercise may also be associated with an increase in reactivity, and the increased ability to form aggregates (ASSENZA et al., 2015). Exercise, especially in young horses at the beginning of the training season activates production of factors responsible for clotting (ASSENZA et al., 2013).

The body of a show-jumping horse is mobilized to draw energy from both alternating aerobic and anaerobic conditions. Properly conducted training should develop both aerobic capacity and the ability to work in conditions of acidity (FAZIO et al., 2014; JAWOR et al., 2007). With an over- intensive workout, there is a risk of damage to the loco-motor system, leading to the elimination of the horse from further performance. According to FAZIO et al. (2014) an increase in AST and CK may be associated with an increase in the permeability of the mitochondrial membrane, and an increase in the size of the liver during training. This phenomenon stems from the moderate increase in all the enzymes involved in the work of muscles (PICCIONE et al., 2010). The activity of AST and $\mathrm{CK}$ after exercise was moderate in the present study, which may indicate a transient increase in the permeability of the myocytes. Due to the varying stimulation of the motor system between workouts, and the different sporting categories of the two groups, the AST increase was greater in group A at the beginning and in the middle of the season. With the end of the training season, horses from group B had lower resting values of AST and lower increases occurred after training in comparison to group A. Similar trends occurred in the resting value of CK activity. It should be added that horses subjected to training (group B) competed in competitions more often, and in increasingly higher level and more difficult competitions throughout the season. The attenuation of CK response to exercise was related to lower extra-fibrilar GLU utilization with LA formation and greater fat metabolism (MUÑOZ et al., 2002). These relationships were partly confirmed in our study (group B). Changes in the activity of AST and CK after rest indicate that the training in both groups was not intense enough to cause muscle damage (SOARES et al., 2011).

The activity of enzymes $\mathrm{CK}$ and $\mathrm{LDH}$ is bound inside the muscle energy processes (REED et al., 2004). The concentration of these two enzymes in the course of the training season showed an upward trend after exercise and then return to the values similar to the resting ones. A significant increase $(\mathrm{P}<0.01)$ of $\mathrm{CK}$ was found at the beginning of 
R. Kupczyński et al.: The influence of different workload trainings on some blood parameters in show jumping horses

the season in Group A after exercise (296.57 U/L). Also, the activity of CK-resting in this group was higher at the end of the season. These activities may indicate that some muscles could become marginally damaged (OSTASZEWSKI et al., 2012). In groups $\mathrm{A}$ and $\mathrm{B}$ there was an increase $(\mathrm{P}<0.01)$ in activity after training in the middle of the season. In group A, the highest increase in the activity related to training took place in the series IIItests, and group B in the series II tests. At the same time resting values of LDH activity in group B increased steadily during the training season, and a similar trend was noted by FAZIO et al. (2014). The activity of CK and LDH may be dependent on the levels of protein, amino acids, and vitamin E in the feed (UPAGANLAWAR et al., 2010). Additional supplementation of these components during the training season reduces the activity of these enzymes, thereby reducing muscle damage. The increase in creatinine levels after exercise may be associated with the increased use of muscle phosphocreatine for creatinine synthesis. After a period of rest, there was a downward trend in the concentration of creatinine (PICCIONE et al., 2010). This was also observed in the present study. Also in Group B, especially at the end of the training season, there was a smaller increase in $\mathrm{K}$ concentrations and then a return to normal resting values. Such changes have been observed in horses during training (PICCIONE et al., 2007), and even after the initial phase of training (FAZIO et al., 2014). The rate of K reduction during recovery is important because the cation is involved in osmosis and the normal balance of water and cellular biochemical functions (FAZIO et al., 2014).

Depending on the discipline, the type of effort, the type of muscle fibers working and, above all, the condition of the body, exertion level LA increases. Lactate concentration in the blood is a marker of anaerobic and aerobic thresholds during exercise in horses (ADAMU et al., 2014). The limit of anaerobic and aerobic thresholds amounting to 4 mmol/L (LINDNER et al., 2009) during exercise may have lower values for specific sports (KUPCZYŃSKI and ŚPITALNIAK, 2015). In both groups the LA concentration showed an increase after training, while in Group B, where additional physical stimulation was present, LA was lower than the resting values at the end of the sporting season. The LA levels obtained in our study indicate that the training in both groups had the character of aerobic training. In the case of exercise endurance (aerobic), the lower concentration of lactate in the blood may indicate the better adaptation of the organism to physical work. With this kind of effort, the faster transport of lactic acid to the muscles and its oxidation occur. Other studies have found that horses with higher blood lactate values after jumping showed decreased show jumping performance and were more likely to display muscle soreness (ROBERTS et al., 2014). Effort exceeding the threshold of the maximum effort, the so-called aerobic capacity or supra-maximal, causes an increase in LA concentration in the blood (ADAMU et al., 2014). This effort is highest during competitions. 
The duration of exercises and their intensity varies and so does the growth of key physiological parameters. This was confirmed in studies conducted on riding horses in swimming training (KANG et al., 2012). During intensive exercise there is an increase in the activity of the hormones responsible for energy metabolism, that is, the catecholamines, and glucagon (FERRAZ et al., 2010). During exercise, mobilization of liver glycogen stores helps to maintain blood glucose concentrations. According to FAZIO et al. (2014) glucose levels may increase after intense exercise due to the increased mobilization of energy from the fat metabolism. Prolonged exercise in endurance horses caused an increase $(\mathrm{P}<0.01)$ in glucose levels after exercise (ADAMU et al., 2012). GEOR et al. (2002) found that 6 weeks of moderate-intensity training resulted in a decrease in glucose flux during submaximal exercise. In our study, smaller changes in GLU concentrations were found after training in Group B, and they also returned more quickly to the resting values.

\section{Conclusion}

Our results indicate that higher resting values at the end of the training season of hematological parameters such as RBC, HGB and HCT indicate the better retraining of horses from group $\mathrm{B}$, using regular exercise on a treadmill. Horses subjected to additional training (group B), which were more frequently involved in tougher competition at the end of the season, had lower activity of AST, LDH and CK than horses not stimulated by further exercises between workouts. In addition, the increase in these enzymes after training was not as clear as in group A. At the end of the sporting season, LA concentrations were lower in group B after training, as well as the lower resting values. In planning equine workouts the treadmill has a significant effect on blood parameters, health status and the degree of training of show-jumper horses. The research indicates the desirability of introducing additional stimulation of movement of horses between the relevant trainings, involving the rider.

\section{Acknowledgements}

This study was financed through the statutory activity of Department of Environment Hygiene and Animal Welfare, Wroclaw University of Environmental and Life Sciences no. B010/0003/15. The publication is supported by the Wroclaw Centre of Biotechnology, a program by the Leading National Research Centre (KNOW) for 2014-2018.

\section{References}

ADAMU, L., N. M. ADZAHAN, A. RASEDEE, B. AHMAD (2014): Responses of serum biochemical parameters, electrolytes and heart rate in an $80 \mathrm{~km}$ endurance race. J. Vet. Adv. 4, 329-337. 
R. Kupczyński et al.: The influence of different workload trainings on some blood parameters in show jumping horses

ADAMU, L., M. A. NORANIZA, A. RASEDEE, A. BASHIR (2012): Changes in biochemical, hematological and cytokinesin endurance horses with metabolic crises. J. Anim. Vet. Advan. $11,3431-3436$

ADAMU, L., M. A. NORANIZA, A. RASEDEE, A. BASHIR (2013): Effect of age and performance on physical, hematological, and biochemical parameters in endurance horses. J. Equine Vet. Sci. 33, 415-420.

ASSENZA, A., F. CONGIU, C. GIANNETTO, F. FAZIO, G. PICCIONE (2015): Haematological response associated with repeted show jumping competition in horse. Acta Sci. Vet. 43, 1305.

ASSENZA, A., F. TOSTO, S. CASELlA, F. FAZIO, C. GIANNETTO, G. PICCIONE. (2013): Changes in blood coagulation induced by exercise training in young athletic horses. Res. Vet. Sci. 95, 1151-1154.

BIS-WENCEL, H., K. LUTNICKI, A. Z. ROWICKA, B. NOWAKOWICZ-DĘBEK, M. BRYL. (2012): Effort of varying intensity as a factor influencing the variability of selected biochemical blood parameters of jumping horses. Bulletin of the Veterinary Institute in Pulawy 56, 225 229.

CASELLA, S., D. ALBERGHINA, C. GIANNETTO, G. PICCIONE (2012): Responses to training and standardised exercise test in the athlete horse: changes in blood gas profile. Comp. Clin. Pathol. 21, 611-614.

FAZIO, F., A. ASSENZA, F. TOSTO, F. CASELLA, G. PICCIONE, G. CAOLA (2011): Training and haematolochemical profile in Thoroughbreds and Standardbreds: A longitudinal study. Livest. Sci. 141, 221-226.

FAZIO, F., V. MESSINA, S. CASELlA, C. GIANNETTO, S. MARAFIOTI, G. PICCIONE (2012): Effect of a stimulate show jumping competition on the blood gas profile of horses trained for show jumping. Turk. J. Vet. Anim. Sci. 36, 259-265.

FAZIO, F., S. CASELLA, A. ASSENZA, F. ARFUSO, F. TOSTO, G. PICCIONE (2014): Blood biochemical changes in show jumpers during a simulated show jumping test. Vet. arhiv 84, 143-152.

FERRAZ, G. C., O. A. B. SOARES, N. S. B. FOZ, M. C. PEREIRA, A. QUEIROZ-NETO (2010): The workload and plasma ion concentration in a training match session of high-goal (elite) polo ponies. Equine Vet. J. Suppl. 42, 191-195.

GEOR, R. J., L. J. MCCUTCHEON, K. W. HINCHCLIFF, R. A. SAMS (2002): Training-induced alterations in glucose metabolism during moderate-intensity exercise. Equine Vet. J. Suppl. 34, 22-28.

JAWOR, P., T. STEFANIAK, J. BORKOWSKI, M. PRZEWOŹNY, H. WIERZBICKI, J. H. SWAGEMAKERS (2007): Utility of selected blood parameters in monitoring the accuracy of show jump-horse training. Med. Weter. 63, 213-218.

KANG, O. D., Y. C. RYU, Y. M. YUN, M. S. KANG (2012): Physiological changes in Jeju crossbred riding horses by swim training. Asian Austral. J. Anim. Sci. 25, 200-206. 
R. Kupczyński et al.: The influence of different workload trainings on some blood parameters in show jumping horses

KUPCZYŃSKI, R., K. ŚPITALNIAK (2015): Analysis of acid-base balance as well as hematological and biochemical parameters in horses of combined driving discipline. Arch. Anim. Breed. 58, 221-228.

LINDNER, A., R. A. LÓPEZ, E. DURANTE, V. FERREIRA, F. M. BOFFI FEDERICO (2009): Conditioning horses at v10 3 times per week does not enhance v4. Equine Vet. Sci. 29, 828832.

LINDNER, A., S. WÄSCHLE, H. H. L. SASSE (2012): Physiological and blood biochemical variables in horses exercising on a treadmill submerged in water. J. Anim. Physiol. Anim. Nutrition. 96, 563-569.

MUÑOZ, A., C. RIBER, R. SANTISTEBAN, R. G. LUCAS, F. M. CASTEJON (2002): Effect of training duration and exercise on blood-borne substrates, plasma lactate and enzyme concentrations in Andalusian, Anglo-Arabian and Arabian breeds. Equine Vet. J. Suppl. 34, 245-251.

NANKERVIS, K. J., R. J. WILLIAMS (2006): Heart rate responses during acclimation of horses to water treadmill exercise. Equine Vet. J. Suppl. 36, 110-112.

OHMURA, H, A. MATSUI, T. HADA, J. H. JONES (2013): Physiological responses of young thoroughbred horses to intermittent high-intensity treadmill training. Acta Vet. Scand. 55, 59.

OSTASZEWSKI, P., A. KOWALSKA, E. SZARSKA, P. SZPOTANSKI, A. P. CYWINSKA, B. BAŁASINSKA, T. SADKOWSKI (2012): Effects of ß-hydroxy-ß-methylbutyrate and oryzanol on blood biochemical markers in exercising Thoroughbred race horses. J. Equine Vet. Sci. 32, 542-551.

PICCIONE, G., C. CASELLA, C. GIANETTO, V. MESSINA, V. MONTEVERDE, G. CAOLA, S. GUTTADAURO (2010): Haematological and haematochemical responses to training and competition in Standardbred horses. Comp. Clin. Pathol. 19, 95-101.

PICCIONE, G., C. GIANNETTO, F. FAZIO, S. DI MAURO, G. CAOLA (2007): Haematological response to different Workload in jumper horses. Bulg. J. Vet. Med. 10, 21-28.

PICCIONE, G., I. VAZZANA, C. GIANETTO, M. GIANESELLA, V. FERRANTELLI (2008): Modification of some haematological and haematochemical parameters in horse during long distance rides. J. Vet. Sci. 1, 37-43.

REED, S. M., W. M. BAYLY, D. C. SELLON (2004): Equine Internal Medicine, $2^{\text {nd }}$ ed., WB Saunders, Philadelphia, pp. 308-312.

ROBERTS, C., P. HARRIS, R. MURRAY, R. CNOCKAERT (2014): The relationship between blood lactate, serum muscle enzymes, jumping performance and muscle soreness in showjumping horses. Equine Vet. J. 46, S46, 9-9.

SOARES, J. C. M., R. ZANELlA, C. BONDAN, L. P. AlveS, R. M. DE LiMA, A. C. DA MOTTA, E. L. ZANELLA (2011): Biochemical and antioxidant changes in plasma, serum, and erythrocytes of horses before and after a jumping competition. J. Equine Vet. Sci. 31, 357-360.

TRIGO, P., F. CASTEJON, C. RIBER, A. MUÑOZ. (2010): Use of biochemical parameters to predict metabolic elimination in endurance rides. Equine Vet. J. 42, 142-146. 
R. Kupczyński et al.: The influence of different workload trainings on some blood parameters in show jumping horses

UPAGANLAWAR, A., H. GANDHI, R. BALARAMAN (2010): Effect of vitamin E alone and in combination with lycopene on biochemical and histopathological alterations in isoproterenolinduced myocardial infarction in rats. J. Pharmacol. Pharmacother. 1, 24-31.

ZOBBA, R., M. ARDU, S. NICCOLINI, F. CUBEDDU, C. DIMAURO, P. BONELLI, C. DEDOLA, S. VISCO, M. L. P. PARPAGLIA (2011): Physical, hematological, and biochemical responses to acute intense exercise in polo horses. J. Equine Vet. Sci. 31, 542-548.

Received: 13 May 2017

Accepted: 18 September 2017

\section{KUPCZYŃSKI, R., K, ŚPITALNIAK, A. ZWYRZYKOWSKA-WODZIŃSKA, M. SOROKO: Utjecaj različitog opterećenja treningom na neke pokazatelje u krvi konja za preponsko jahanje. Vet. arhiv 88, 279-293, 2018.}

\section{SAŽETAK}

Cilj istraživanja bio je procijeniti promjene u hematološkim i biokemijskim pokazateljima kod konja za preponsko jahanje. Konji su podvrgnuti različitim opterećenjima treningom: skupina A trenirala je s jahačem i bez dodatnih vježbi, skupina B imala je dodatan dnevni trening na pokretnoj traci za trčanje. Uzorci krvi uzeti su u mirovanju, prije vježbanja i 24 sata nakon natjecanja u preponskom jahanju (početak, sredina i završetak sezone). Bez obzira na vrstu treninga te razinu njegova intenziteta i opterećenja, nakon vježbanja ustanovljeno je povećanje broja eritrocita (RBC), koncentracije hemoglobina (HGB) i hematokrita (HCT). U skupini B došlo je do povećanja $(\mathrm{P}<0,05)$ pokazatelja u mirovanju: $\mathrm{RBC}$ i HGB između početka sezone i sredine sezone. Tijekom istraživanja u obje je skupine nakon treninga došlo do povećanja aspartat-aminotransferaze (AST), laktat dehidrogenaze (LDH) i kreatin kinaze (CK). Konji iz skupine B, podvrgnuti dodatnom treningu, na kraju sezone imali su nižu aktivnost pokazatelja AST, LDH i CK u mirovanju od konja iz skupine A. U skupini B pokazatelji mliječne kiseline (LA) u mirovanju bili su niži u sredini i na kraju sportske sezone u usporedbi sa skupinom A. Istraživanje upućuje na poželjnu praksu kojom se između treninga, koji uključuju jahača, uvodi dodatna stimulacija kretanja konja.

Ključne riječi: preponsko jahanje; metode treninga; krvni pokazatelji; pokretna traka za trčanje 
